J. Lake Sci.(湖泊科学), 2016, 28(4): 875-886

DOI 10. 18307/2016. 0422

(C) 2016 by Journal of Lake Sciences

\title{
长江中下游典型湖泊营养盐历史变化模拟”
}

\author{
郭 娅, 于 革** \\ ( 中国科学院南京地理与湖泊研究所湖泊与环境国家重点实验室,南京 210008)
}

\begin{abstract}
摘 要: 湖泊营养盐变化在自然条件下受到气候水文因素控制, 同时受到湖泊生态系统生物群落作用和反馈. 作为动力 机制探讨, 本文试图基于水文和生态动力学方法, 分别构建气候-流域水文作用于湖泊营养盐的外源模式和湖泊生物群落 作用于湖泊营养盐的內源模式. 针对长江中下游典型湖泊, 经过控制实验和率定, 发现营养盐模拟与观测数据在时间序 列上达到 90\%百分位的正相关,因此用来模拟 1640-1840 A.D. 期间的营养盐演变历史. 研究表明: (1) 模拟的湖泊营养 盐变化与沉积钻孔揭示的历史营养盐变化基本一致, 沉积记录与模式模拟的 7 个湖泊的营养盐变化均显著相关; (2)气候 因素是湖泊营养盐历史演变的主控因子, 来自于湖泊生物群落的反馈作用贡献约占 $40 \%$; (3) 在温度和降水因子的驱动下, 湖泊营养盐历史变化主要受降水控制, 在极端干旱时期, $60 \%$ 的营养盐变化同步响应于降水变化. 同时, 面积在 $400 \mathrm{~km}^{2}$ 以下 的湖泊营养盐对气候变化的响应比 $2000 \mathrm{~km}^{2}$ 以上的大湖更为敏感. 研究结果对长江中下游湖泊营养状态的长期变化机 理认识和趋势控制提供科学依据.
\end{abstract}

关键词: 气候驱动;湖泊生态系统;生物量;模拟; 总磷; 长期变化

\section{Simulations on historical nutrient changes of typical lakes in the middle and lower reaches of Yangtze River, China}

\section{GUO Ya \& YU Ge**}

( State Key Laboratory of Lake Science and Environment, Nanjing Institute of Geography and Limnology, Chinese Academy of Sciences, Nanjing 210008, P.R.China)

\begin{abstract}
The natural process of lake trophic status is controlled by both climatic-hydrological factors and ecosystem interactions. In order to recognize the response mechanism of lake nutrients to the multiple factors, 2 models were designed to simulate the lake nutrient changes over 1640-1840 A.D. in some lakes in the Yangtze floodplain. One was an exogenous-forced model with external driving forces from climate and catchment hydrological changes; the other was an endogenous-forced model with internal driving forces from the biological interactions within the lake ecosystem. After approval test and calibration, the simulation results by the 2 models had significant positive relationships with the observational records in 90th percentile, indicating robust simulation on the historical nutrient changes in 1640 -1840 A.D. Results showed that (1) the simulated total phosphorus series by the combined exogenous-and endogenous-forced model were consistent with the reconstructed total phosphorus series from sediment cores in the 7 lakes in the Yangtze floodplain; (2) climate change was the primary factor for the long-term lake nutrient changes, whereas the feedback effects from lake ecosystem accounted for $40 \%$ of the nutrient changes; (3) the long-term changes of lake nutrients were significantly related with precipitation with $60 \%$ of the historical nutrient changes in synchronous response to precipitation changes. In addition, the responses of TP variations in the lakes smaller than $400 \mathrm{~km}^{2}$ were more sensitive to climate changes than that in the large lakes over $2000 \mathrm{~km}^{2}$.
\end{abstract}

Keywords: Climate change; lake ecosystem; biomass; simulation; total phosphorus; long-term

近几十年来全球变化加速水体生态系统重大变化 ${ }^{[1]}$. 在强烈人类干扰背景下, 气温与降水的变化可以

* 科技部全球变化重大科学研究计划 (2012CB956103) 和中国科学院创新团队国际合作伙伴计划项目 ( KZZD-EWTZ-08) 联合资助.2015-01-23 收稿; 2015-10-14 收修改稿. 郭娅( 1982 ), 女, 博士研究生; E-mail: yguo@ niglas. ac.cn.

** 通信作者;E-mail:geyu@ niglas.ac.cn. 
直接或间接地影响磷循环, 进而使湖泊水生态系统受到影响 ${ }^{[2]}$. 这些影响包括沉水植物消亡、蓝藻水华频 发、微生物生产力增加、生物多样性下降、营养盐的循环与利用效率加快等 ${ }^{[3]}$. 从流域来看, 气候变暖促进湖 泊接受更多的营养物质输人、加速湖泊的富营养化 ${ }^{[4]}$. 这些基于观测尺度的研究, 阐明了湖泊营养盐对气候 变化和生态系统响应的特点, 但这些结论能否解释湖泊营养状态的长期演化, 目前还缺少系统认识.

长江中下游地处中国经济发达区, 也是中国淡水湖泊最为集中的区域, 目前该区域绝大多数湖泊已经 富营养化或正处于富营养化中 ${ }^{[5]}$. 然而, 在当前气温上升、人为活动急剧加重的背景下, 该区域湖泊营养状 态的演化机理更为复杂, 难以分辨和认识. 由于缺乏长期过程的监测, 人类活动干扰前这些湖泊生态系统的 自然演变过程、目前湖泊的营养状态以及发展趋势如何并不清楚, 而了解这些信息对湖泊治理和修复方案 的目标制定具有重要的参考价值. 国内外关于长江中下游湖泊富营养化的演变过程已经开展了较多研 究 ${ }^{[6-8]}$, 但大多局限在季节、年际等短尺度上, 即使年际尺度的研究也不超过数十年; 依赖于湖泊沉积物微体 化石信息的长尺度研究, 受制于研究材料的获取难度及精度的影响, 难以推广到大量湖泊中, 制约了对湖泊 生态系统演化过程和机制的认识. 而采用物理-生态学模型对尚未发生的过程进行动力学机制的模拟和预 测, 能够突破时间限定反演时空演变并预测湖泊生态系统的动态变化.

1840 年以前长江中下游处于自然的农耕时代, 人为活动干扰强度低 ${ }^{[9]}$, 能够提供认识湖泊响应气候的 参照系统. 为此, 本文针对湖泊生态系统在气候变化下的响应, 选择自然农耕时期 (1640-1840 A.D.) 为研 究时段, 以气候变化为驱动因子, 流域水文变化为限制因子 ${ }^{[10]}$, 并以湖泊营养盐变化作为状态变量, 试图构 建流域湖泊营养盐-气候驱动的 “外源” 模型 (模型 1 : 湖泊营养盐赋存对气候-水文响应模型) 和湖泊生态反 馈-营养盐变化的“内源” 模型 (模型 2: 湖泊主要生物群落生物量与营养盐关系模型), 通过对比外源 (流域 气候、水文、地貌) 和内源 (沉积、生物和生态系统) 不同层次动力下两种模式的营养盐输出, 获得在外源和内 源影响下湖泊营养盐变化的特征和差异, 揭示不同影响因素对湖泊营养盐作用的动力机制. 本文的模型应 用到长江中下游太湖等 8 个湖泊, 并采用湖泊钻孔资料重建的营养盐变化特征对模拟结果进行验证.

\section{1 研究区概况}

长江中下游地区湖泊密布, 根据 2000 年全国第二次湖泊调查结果, $1 \mathrm{~km}^{2}$ 以上的湖泊 651 个, 面积 16558 $\mathrm{km}^{2}$, 占全国淡水湖泊面积的 $60 \%$; 面积大于 $10 \mathrm{~km}^{2}$ 的湖泊 136 个 $^{[11]}$ (图 1). 这些湖泊历史上均与长江自然 连通,形成了自然的江、河、湖复合生态系统, 且与江、河水情息息相关. 从成因类型上看,这些湖泊大多属于 河间洼地湖 (如洪湖)、隹塞湖 (如龙感湖)、沉溺湖 (如网湖) 等, 也有构造断陷形成的湖泊 (如鄱阳湖), 并集 中分布在中、下游 4 个构造抝陷和沉降洼地区, 由此形成了洞庭-江汉、华阳-鄱阳和太湖-三角洲 3 大湖群. 在相同的自然地理环境背景下, 研究区湖泊具有以下共同特征: (1) 湖水深度 $2 \sim 5 \mathrm{~m}$ 不等, 湖底平坦, 属于 浅水型湖泊. (2) 受长江水位和降水影响, 湖泊水位在年内变化大, 少数湖泊历史最高水位可达 $10 \mathrm{~m}$ 以上. 由此造成岸线不稳, 丰水位和枯水位期间湖面发生明显扩展和缩小. (3) 湖底淤泥深厚, 有机质含量高. 主要 为近现代的湖相沉积, 淤泥厚度大多在 1 2 m, 部分湖泊 (如洪湖、太湖) 可达 $3 \mathrm{~m}$, 湖泥有机质含量高达 $2 \% \sim$ $6 \%{ }^{[12]}$. (4) 从长江洪泛平原第四纪松散沉积构造来看, 这些湖相沉积与冲积物构成交互层理. 快速沉积的 河床相沙砾层与湖沼相黏土和淤泥层呈现出多个交替、旋回的沉积构造 ${ }^{[12]}$.

依据长江中下游湖泊的面积、营养状况以及生态类型等条件, 在该研究区内选取太湖、洞庭湖、鄱阳湖 作为面积大于 $2000 \mathrm{~km}^{2}$ 的大型浅水湖泊, 巢湖、石臼湖、洪湖、龙感湖作为面积在 200 $800 \mathrm{~km}^{2}$ 的中型浅水湖 泊, 以及小于 $50 \mathrm{~km}^{2}$ 的小型浅水湖泊网湖 (图 1, 表 1), 作为典型湖泊进行历史时期营养盐变化的模拟研究.

\section{2 资料收集与处理}

\section{1 气候数据}

2.1.1 现代气候数据 根据长江中下游地区 59 个国家一级气象站 1951-2000 年逐月温度和降水数据 ${ }^{[14]}$ 及 气象站位置, 以半径小于 $60 \mathrm{~km}$ 内插到 8 个典型湖泊点上, 获得 8 个湖泊逐月温度和降水资料. 逐月潜在蒸 散发 (PET) 采用 Thornthwaite 法 ${ }^{[15]}$, 根据多年的逐月温度计算饱和水汽密度经验公式获得.

2.1.2 历史气候数据 由于缺少气候观测, 历史气候数据采用 GCM 模拟资料. 采用德国 ECHAME5 全球气候 
模型在 IPCC-CMIP5 框架下的模拟结果 ${ }^{[16]}$, 并采用统计降尺度法获得太湖等 8 个湖泊 1640 - 1840 A.D.

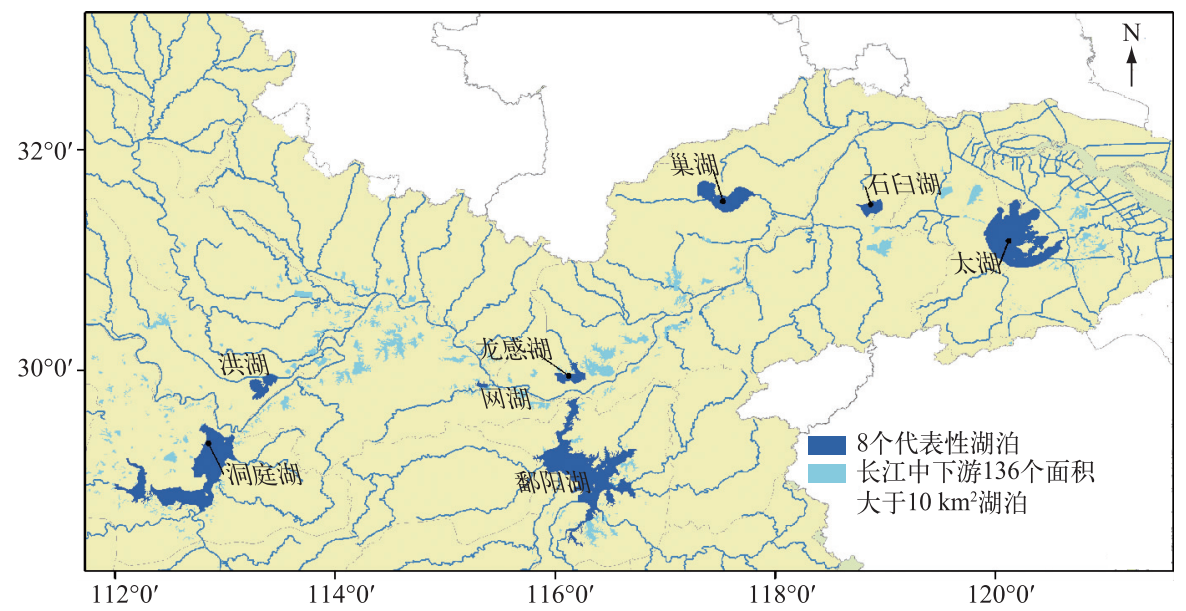

图 1 长江中下游 8 个典型湖泊分布

Fig. 1 Locations of the 8 typical lakes in the middle and lower reaches of Yangtze River

表 1 典型湖泊的地理、水文、营养和生态信息

Tab.1 Geographic, hydrological and biological information of the 8 typical lakes

\begin{tabular}{llcccccc}
\hline 湖泊名称 & 经纬度及海拔高度 & $\begin{array}{c}\text { 面积/ } \\
\mathrm{km}^{2}\end{array}$ & $\begin{array}{c}\text { 流域面积/ } \\
\mathrm{km}^{2}\end{array}$ & $\begin{array}{c}\text { 容量/ } \\
\left(\times 10^{8} \mathrm{~m}^{3}\right)\end{array}$ & $\begin{array}{c}\text { 平均深度/ 总磷 }(\mathrm{TP}) / \\
\mathrm{m} \\
(\mathrm{mg} / \mathrm{L})\end{array}$ & $\begin{array}{c}\text { 草/藻 } \\
\text { 占优势 }[5,13]\end{array}$ \\
\hline 太湖 & $31.25^{\circ} \mathrm{N}, 120.23^{\circ} \mathrm{E} ; 3.06 \mathrm{~m}$ a.s.l. & 2425.0 & 36500 & 51.40 & 2.12 & 0.133 & 藻 \\
石臼湖 & $31.47^{\circ} \mathrm{N}, 118.85^{\circ} \mathrm{E} ; 10.00 \mathrm{~m}$ a.s.l. & 210.4 & 18600 & 8.58 & 4.08 & 0.060 & 草 \\
巢湖 & $31.57^{\circ} \mathrm{N}, 117.57^{\circ} \mathrm{E} ; 8.15 \mathrm{~m}$ a.s.l. & 753.0 & 9258 & 20.70 & 2.69 & 0.175 & 藻 \\
洪湖 & $29.81^{\circ} \mathrm{N}, 113.25^{\circ} \mathrm{E} ; 25.00 \mathrm{~m}$ a.s.l. & 344.4 & 10352 & 6.58 & 1.90 & 0.046 & 藻 \\
网湖 & $29.87^{\circ} \mathrm{N}, 115.35^{\circ} \mathrm{E} ; 7.30 \mathrm{~m}$ a.s.l. & 42.3 & 5310 & 1.57 & 3.70 & 0.060 & 草 \\
龙感湖 & $29.97^{\circ} \mathrm{N}, 116.13^{\circ} \mathrm{E} ; 12.00 \mathrm{~m}$ a.s.l. & 316.2 & 5511 & 11.96 & 3.78 & 0.077 & 草 \\
洞庭湖 & $29.33^{\circ} \mathrm{N}, 112.83^{\circ} \mathrm{E} ; 24.30 \mathrm{~m}$ a.s.l. & 2740.0 & 257000 & 155.44 & 6.39 & 0.030 & 草 \\
鄱阳湖 & $29.08^{\circ} \mathrm{N}, 116.33^{\circ} \mathrm{E} ; 14.01 \mathrm{~m}$ a.s.l. & 2933.0 & 162000 & 149.60 & 5.10 & 0.070 & 草 \\
\hline
\end{tabular}

逐月温度和降水序列. 于革等采用了鄱阳湖历史文献和考古调查的史料对 ECHAM5 气候模拟结果予以验 证, 表明 ECHAM5 模拟 1640-1840 A.D. 的降水变化可以接受 ${ }^{[16]}$.

\section{2 湖泊营养盐数据}

2.2.1 现代营养盐观测数据 8 个湖泊营养盐逐年调查资料根据文献收集 ${ }^{[5,17-24]}$. 此外, 2 次系统性资料来自 于区域性湖泊调查: (1) 1980-1990s 开展了第一次长江中下游湖群水体营养盐的调查, 表明该阶段的湖泊 水体总磷浓度在 $0.019 \sim 0.268 \mathrm{mg} / \mathrm{L}$ 之间 ${ }^{[11]}$; (2) 1998- 2000 年开展的第二次湖泊调查, 表明长江中下游区 域已无贫营养化湖泊, 中营养化湖泊仅占 $6 \%$, 中富营养化湖泊占 $45 \%$, 富营养化湖泊占 $48 \%$, 湖泊的总磷浓 度在 $0.035 \sim 0.268 \mathrm{mg} / \mathrm{L}$ 之间 ${ }^{[19]}$. 尽管该时期水体营养盐状态显著受到流域排放和污染影响, 本文仅利用湖 泊总磷浓度与气候水文、湖泊生态系统的响应关系进行控制实验的参数设置.

2.2.2 历史营养盐数据 历史时期湖泊的营养盐状况主要通过湖泊钻孔岩芯、采用古湖沼学的湖泊沉积物计 年技术和湖泊营养状况指示信息的测试技术获得 ${ }^{[25]}$. 根据太湖 ${ }^{[21]}$ 、石臼湖 ${ }^{[22]}$ 、巢湖 ${ }^{[23]} 、$ 洪湖 ${ }^{[24]}$ 、网湖 ${ }^{[26]}$ 、 龙感湖 ${ }^{[27]}$ 及鄱阳湖的沉积钻孔研究, 本文进行编辑整理, 建立这些湖泊营养盐变化的历史档案 (图 2), 其中 由于洞庭湖钻孔没有历史营养盐相关资料故未在图中列出. 尽管沉积物中的总磷与水体总磷的量值和量级 有差别, 但大量的古湖沼研究证明许多沉积物中的总磷变化能够反映过去湖泊水体总磷的变化 ${ }^{[28-29]}$, 因此 
可用来对历史时期的总磷变化模拟进行验证.

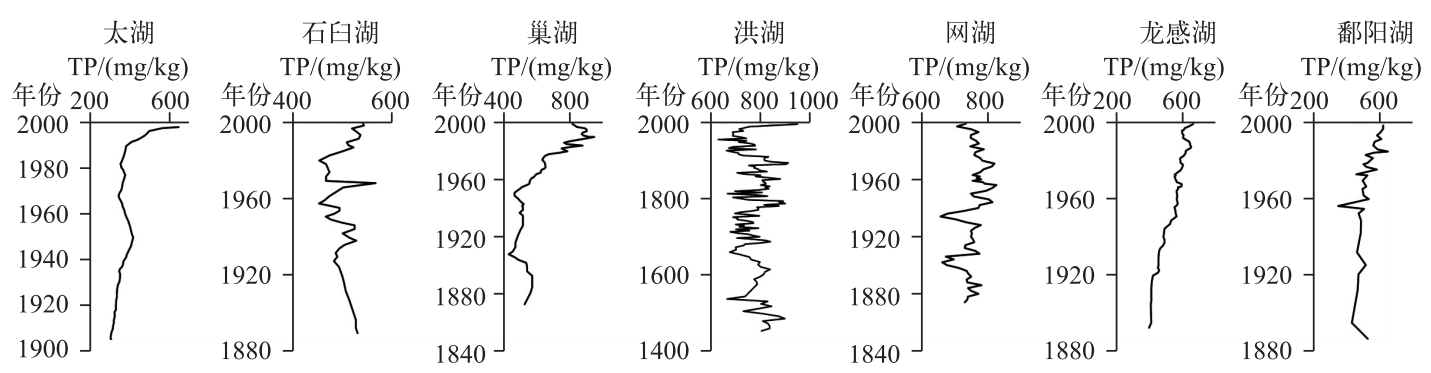

图 2 长江中下游 7 个典型湖泊钻孔沉积物总磷含量变化序列

Fig.2 Dating results and proxy-TP contents series in the sediment cores of the 7 lakes in the middle and lower reaches of Yangtze River

\section{3 湖泊水文数据}

根据恒定流理论 ${ }^{[30]}$, 径流量 $(W)$ 为单位时间内径流深度 $(R)$ 与汇流面积 $\left(A_{c}\right)$ 乘积. 径流深度 $R$ 是单位 时间的径流总量平铺在流域面积上所得的水层厚度, 对于汇人湖泊的流域而言, 可以通过流域降水量与蒸 发的水量平衡方程 $R=P-E$ 估算. 其中 $W$ 反映了流域输人湖泊的平均来水情况, 是湖泊水量可能的最大 限度.

$$
W=(P-E) A_{c}
$$

式中, $P 、 E$ 分别是降水量和蒸发量. 可根据长江中下游湖泊观测的多年降水平均值获得 $P$, 计算潜在蒸发获 得 $E$, 以及流域面积 $\left(A_{c}\right)$ 估算出年流域人湖径流量. 由于气象站不在湖泊中心位置, 理论计算径流量由于温 度、降水以及潜在蒸散发有系统误差, 与观测值有一定差距. 根据长江中下游多个湖泊 (固城湖、洪湖、梁子 湖、巢湖、太湖、石臼湖、鄱阳湖、洞庭湖) 长期水文观测数据和统计资料, 发现湖泊观测值与模拟值呈线性相 关, 可达 $98 \%$ 的置信域. 因此,采用关系系数 $\alpha$ 对模拟值进行调整:

$$
W=\alpha(P-E) A_{c}
$$

模式计算的偏差在 $0.34 \sim 1.00$ 之间变化,70\%的湖泊 $\alpha$ 值大于 0.60 .

\section{4 湖泊初级生产者与顶级掠食者生物量数据}

湖泊生态系统内部和生物群落间的相互作用影响其营养盐利用的效率, 进而影响水体中营养物质的含 量. 假定湖泊主要生物群落的生态学特征在生态系统长期变化过程中保持相对稳定, 本文以位居湖泊生态 系统的初级生产者 (包括浮游植物和高等水生植物群落)、顶级生物群落 (以湖泊生物链的顶级掠食者鱼类 群落代表) 的生物量代表湖泊生态系统起点和终端的生物量初始值, 来反映生态系统特征的变化 ${ }^{[31]} .8$ 个湖 泊的水生植物、藻类生物量及鱼类捕获量 (表 2) 来自于 $1980 \mathrm{~s}$ 对太湖等 8 个湖泊的生态调查资料 ${ }^{[11]}$.

表 28 个湖泊藻类、水生植物及鱼类群落生物量

Tab.2 Biomass of the major aquatic communities in the 8 lakes

\begin{tabular}{lcccccccc}
\hline 生物量 & 太湖 & 巢湖 & 龙感湖 & 洪湖 & 网湖 & 洞庭湖 & 石臼湖 & 鄱阳湖 \\
\hline 藻类生物量 $/\left(\times 10^{4} \mathrm{t} / \mathrm{a}\right)$ & 202.3 & 177.1 & 1.8 & 5.2 & 9.7 & 1.2 & 0.6 & 127.9 \\
水生植物生物量 $/\left(\times 10^{4} \mathrm{t} / \mathrm{a}\right)$ & 2.7 & 2.7 & 435.4 & 383.3 & 116.7 & 496.7 & 300.0 & 193.2 \\
鱼类捕获量 $/\left(\times 10^{4} \mathrm{t} / \mathrm{a}\right)$ & 0.4 & 1.2 & 0.5 & 0.8 & 1.0 & 0.5 & 1.6 & 0.9 \\
\hline
\end{tabular}

\section{3 研究方法}

本文构建 2 个湖泊营养盐动力模式: 模式 1 为湖泊营养盐对气候-水文变化的响应模式; 模式 2 在模式 1 的基础上叠加考虑了湖泊生态系统的反馈作用对营养盐变化的影响, 即构建了湖泊生态系统中营养盐与生 物量动力模式. 分别采用这 2 种模式对湖泊营养盐变化过程进行模拟. 


\section{1 湖泊营养盐对气候水文变化响应模式( 模式 1)}

采用径流量对气候变化响应的计算模式 ${ }^{[30]}$ : 假设气候变化下降水 $(P)$ 、蒸发 $(E)$ 和径流 $(Q)$ 的变化量分 别为 $p=P_{1} / P_{0} 、 e=E_{1} / E_{0}$, 和 $q=Q_{1} / Q_{0}(0$ 代表现代值, 1 代表新值), 根据流域水量平衡原理 $(Q=P-E)$ 和径 流系数定义 $(w=Q / P)$, 径流量变化 $(q)$ 对降水变化 $(p)$ 和蒸发变化 $(e)$ 的响应有下列关系式:

$$
q=[p-(1-w) e] / w
$$

在此水量平衡的基础上, 推导湖泊营养盐的变化. 设 $P$ 和 $E$ 为流域降水总量, $R$ 和 $Q$ 分别为进人湖泊和 流出湖泊径流总量, 长期湖泊的水量平衡有: $P+R-E-Q=0$. 设 $C_{\mathrm{p}} 、 C_{\mathrm{r}} 、 C_{1}$ 分别为降水、人流和出流所携带的营 养盐浓度. 若忽略蒸发所消耗的营养盐, 在一定时间 $(t)$ 的湖泊的营养盐变化是:

$$
\mathrm{d}\left[C_{1}(t) V\right] / \mathrm{d} t=C_{\mathrm{p}} P+C_{\mathrm{r}} R-C_{1}(t) Q
$$

式中, $V$ 是湖泊蓄水总量. 当定义营养盐平衡点浓度 $\left(C_{\mathrm{eq}}\right)$ 为营养盐和水量的平衡: $C_{\mathrm{eq}} \equiv\left(C_{\mathrm{p}} P+C_{\mathrm{r}} R\right) / Q$, 根 据流域 $Q=P+R-E$, 其平衡点成为 $C_{\mathrm{eq}}=\left(C_{\mathrm{p}} P+C_{\mathrm{r}} R\right) /(P+R-E)$; 在瞬时时间 $t$ 、时间总量 $T$ 、营养盐浓度初 始量 $C_{1}(0)$ 和营养盐平衡点 $C_{\mathrm{eq}}$ 条件下, (4) 式有解析解: $C_{1}(t)=C_{\mathrm{eq}}+\left[C_{1}(0)-C_{\mathrm{eq}}\right] \exp (-t / T)$.

若设湖泊营养盐浓度 $C_{1}(t)$ 为 $y$, 暂不考虑大气降水中的营养盐浓度, (4) 式可写成一个微分方程:

$$
y^{\prime}=1 / V\left(C_{\mathrm{r}} R-(p+q(p, e)-e) y\right)
$$

其中函数 $q(p, e)$ 采用公式 (3) 计算. 由此可根据湖泊的气象、水文资料, 对长江中下游典型湖泊逐个进 行营养盐浓度变化模拟计算, 模式 1 的营养盐输出单位为 $\mathrm{mg} / \mathrm{L}$.

\section{2 湖泊生态系统中营养盐与生物量动力模式 (模式 2)}

生物量动力模式的核心方程依据 Lotka-Volterra 模型以及扩展的动力模式 ${ }^{[16]}$. 本研究采用湖泊生态系 统中底层初级生产者与顶层掠食者, 设计了生态链两端的概化模式, 试图捕捉在时间尺度上 $10 \sim 10^{2}$ 年湖泊 生态链-生物量主要变化和特征. 湖泊初级生产者主要是具有光合作用的植物,包括浮游微体植物藻类和高 等水生维管束植物. 构建浮游微体植物群落 (简称藻类群落) 与高等水生植物群落 (简称草类群落) 的生态 动力方程, 需要确定两者之间的生态食物链关系和两者状态变量的量级 (生物量). 从生态上看, 大型水生植 物与浮游藻类之间主要是竞争关系: 首先, 他们都是水体初级生产者, 通过对光的竞争以进行光合作用维持 基本的生命过程 ${ }^{[32]}$; 其次, 两者均吸收水体中的营养盐, 构成相互竞争; 第三, 草的分泌物抑制了藻的生

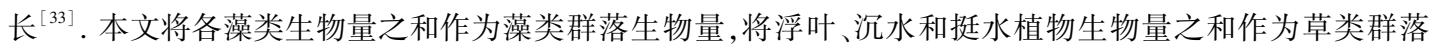
生物量. 作为完整的湖泊生态系统, 本文引人鱼类群落作为湖泊食物链的顶级掠食者. 长江中下游湖泊以鲤 鱼为主, 其次为青鱼、草鱼、鲢鱼、鱅鱼. 鲤鱼、青鱼是底栖杂食性鱼类, 草鱼和鲢鱼主要以草、藻为食. 在这个 系统中, 鱼类与水生植物是捕食者与被捕食者关系, 被捕食者中藻类与草类又是竞争关系. 考虑到生物量在 季节和年际的变化, 根据 SKT 模型原理和结构, 把生物群落随不同时间尺度变化的时间函数 $g(t)$ 增加到模 型中. 由此, 根据 Lotka-Voltera 模型的基本结构, 设 $y_{1}$ 为藻类群落生物量, $y_{2}$ 为草类群落生物量, $y_{3}$ 为鱼类群落 生物量,构成的湖泊生态系统 3 个主要群落生物量对时间变化的常微分方程组 (以下 $y^{\prime}$ 均代表 $\mathrm{d} y(t) / \mathrm{d} t$ ):

$$
\begin{aligned}
& y_{1}{ }^{\prime}=y_{1}\left(a_{1}+a_{2} y_{3}+a_{4} y_{1}+d_{1} g_{1}(t)\right) ; \\
& y_{2}{ }^{\prime}=y_{2}\left(b_{1}+b_{3} y_{3}+b_{4} y_{2}+d_{2} g_{2}(t)\right) ; \\
& y_{3}{ }^{\prime}=y_{3}\left(c_{1}+c_{2} y_{1}+c_{3} y_{2}+c_{4} y_{3}+d_{3} g_{3}(t)\right) .
\end{aligned}
$$

式中, 藻类群落参数 $a$ 、草类群落参数 $b$ 和鱼类群落参数 $c$ 根据 1980-2010 年湖泊生态调查资料, 采用非线 性优化拟合方法获得 ${ }^{[34]}$.

水体营养盐是水生生物的主要限制性资源. 前人总结了不少适用的关系模式来研究水体营养盐浓度对 浮游植物生长、竞争, 以及藻类对营养盐的摄取和吸收, 例如 Micheal-Menten 模式、Monod 模式、Tilman 模式


学式是 Logistic 方程:

$$
N=N_{\mathrm{c}} /\left(k+N_{\mathrm{c}}\right)
$$

式中, $N$ 是藻类生物量的增长量, $N_{c}$ 是水体营养盐浓度, $k$ 是半饱和常数 (Micheal 常数), 相当于藻类增长率 一半时的增长量.

高等水生植物主要摄取湖底沉积物的营养盐, 可采用线性统计关系进行描述 ${ }^{[36]}$ : 


$$
N=d N_{c}
$$

式中, $N$ 是草类生物量的增长量, $N_{\mathrm{c}}$ 是水体营养盐浓度, $d$ 是 $N$ 与 $N_{\mathrm{c}}$ 关系系数. 该统计关系也考虑到鱼类与 营养盐关系.

设 $y_{4}$ 为营养盐状态变量, $d_{1} 、 d_{2} 、 d_{3}$ 分别为藻、草、鱼类生物量增长方程式 (式 (7) 和 (8)) 中关系系数, 3 个生物群落在湖泊中摄取营养盐分别为 $d_{1} y_{4} /\left(k_{1}+y_{4}\right) 、 d_{2} y_{4} 、 d_{3} y_{4}$. 根据方程 (6) 可构成水体营养盐作用在湖 泊群落生物量变化的微分方程:

$$
\begin{aligned}
& y_{1}{ }^{\prime}=y_{1}\left(a_{1}+a_{2} y_{3}+a_{4} y_{1}+d_{1} y_{4} /\left(k_{1}+y_{4}\right) g_{1}(t)\right) ; \\
& y_{2}{ }^{\prime}=y_{2}\left(b_{1}+b_{3} y_{3}+b_{4} y_{2}+d_{2} y_{4} g_{2}(t)\right) ; \\
& y_{3}{ }^{\prime}=y_{3}\left(c_{1}+c_{2} y_{1}+c_{3} y_{2}+c_{4} y_{3}+d_{3} y_{4} g_{3}(t)\right) .
\end{aligned}
$$

同时, 考虑在长江中下游的通江湖泊中, 湖泊积累的营养盐是在湖泊营养盐初始水平上, 流域汇人营养 盐和湖泊生物摄取营养盐的平衡. 根据营养盐在水文气候下流域与湖泊的平衡, 采用 5 式联立 9 式构建湖 泊营养盐变化与湖泊三个群落生物量变化动力关系, 形成 4 联微分方程组:

$$
\begin{aligned}
& y_{1}{ }^{\prime}=y_{1}\left(a_{1}+a_{2} y_{3}+a_{4} y_{1}+d_{1} y_{4} /\left(k_{1}+y_{4}\right) g_{1}(t)\right) ; \\
& y_{2}{ }^{\prime}=y_{2}\left(b_{1}+b_{3} y_{3}+b_{4} y_{2}+d_{2} y_{4} g_{2}(t)\right) ; \\
& y_{3}{ }^{\prime}=y_{3}\left(c_{1}+c_{2} y_{1}+c_{3} y_{2}+c_{4} y_{3}+d_{3} y_{4} g_{3}(t)\right) ; \\
& y_{4}{ }^{\prime}=1 / V\left(C_{\mathrm{r}} R-(p+q(p, e)-e) y_{4}\right) .
\end{aligned}
$$

在微分方程系统中, 设置方程参数、初始值、求解区间, 使用 MATLAB 中常微分方程变步长的求解函数 ODE45, 通过四阶的龙格-库塔算法获得数值解. 由此可根据湖泊的初级生产者与顶级掠食者生物量及湖泊 的气象、水文资料,对长江中下游典型湖泊逐个进行湖泊营养盐负荷变化模拟计算. 模型输出为 3 个群落的 生物量变率, 其矢量 $\left[y_{1}, y_{2}, y_{3}\right]$. 生物量变率 $\left[y_{1}, y_{2}, y_{3}\right]$ 与生物量 $(B)$ 的关系是 $B_{i}=\alpha_{i} \cdot B_{0 i} \cdot y_{i}(i=1,2,3)$, 其中 $B$ 是生物量, $B_{0}$ 是初始生物量. 模型输出第 4 个变量是营养盐变率, 营养盐变率 $\left(y_{4}\right)$ 与营养盐 $(N)$ 的关 系是 $N=\beta \cdot N_{0} \cdot y_{4}$, 其中 $N$ 是营养盐浓度, $N_{0}$ 是初始营养盐浓度. $\alpha$ 和 $\beta$ 是与方程内微分步长、变率有关的 系数.

\section{3 控制实验(1950-2000 A.D.)}

为对模式 1 和模式 2 进行率定和校验, 采用模式 1 即湖泊营养盐对气候/流域径流量变化的响应模式, 模拟长江中下游 8 个湖泊近 50 年来的变化, 采用模式 2 模拟湖泊营养盐在生物量的营养富集下该时段变 化, 并与已有的太湖、巢湖、洪湖营养盐观测数据进行对比 (其余湖泊的观测数据不足). 结果表明, 这 3 个湖 泊采用模式 1 和模式 2 的模拟结果与观测结果在时间序列上达到 $90 \%$ 百分位的正相关, 显示构建模式能够 捕捉到气候水文变化下的湖泊营养盐变化主要特征和过程.

\section{4 结果}

\section{1 湖泊历史营养盐演变模拟}

分别采用模式 1 和模式 2 分别模拟 8 个湖泊 1640-1840 A.D.的营养盐变化序列. 根据模式设置, 模式 1 输出为湖泊营养盐浓度 (量纲为 $\mathrm{mg} / \mathrm{L}$ ), 模式 2 输出为湖泊营养盐负荷 (量纲为 $\mathrm{t} / \mathrm{a}$ ), 为便于 2 种模式的比 较分析,采用模拟结果的距平值 $(\%)$ 来表征其变化过程及特征. 模式 1 中 8 个湖泊的营养盐浓度变化距平 值在 $-13.3 \% \sim+58.5 \%$ 之间变化, 模式 2 中营养盐负荷距平值在 $-44.5 \% \sim+108.3 \%$ 之间变化 (图 4).

此外, 对 2 个模拟结果进行 20 年滑动平均比较各湖泊的营养盐变率 (图 4), 发现模式 1 和模式 2 模拟 结果具有一致性: 8 个湖泊均在 1727-1746 A.D.出现营养盐增加最大值; 营养盐降低最小值出现的时期不 尽一致,巢湖、石臼湖和太湖在 1786-1805 A.D. 营养盐降幅最大, 洪湖和网湖在 1799-1818 A.D. 年营养盐 降幅最大,龙感湖、洞庭湖和鄱阳湖在 $1747-1766$ A.D.年营养盐降幅最大.

\section{2 模拟结果与沉积物指标对比}

根据已有钻孔记录的营养盐变化序列及对应沉积物的高分辨率定年结果, 获得了多个历史年份的沉积物 $\mathrm{TP}$ 值, 将 2 种模式下太湖等 7 个湖泊模拟营养盐与沉积物 TP 指标进行同时期对比. 结果表明,除了模式 1 输 出结果与网湖沉积物指示结果无相关性外, 其它 6 个湖泊均呈显著正相关 $(n=29 \sim 77, P \leqslant 0.1)$; 模式 2 中 7 

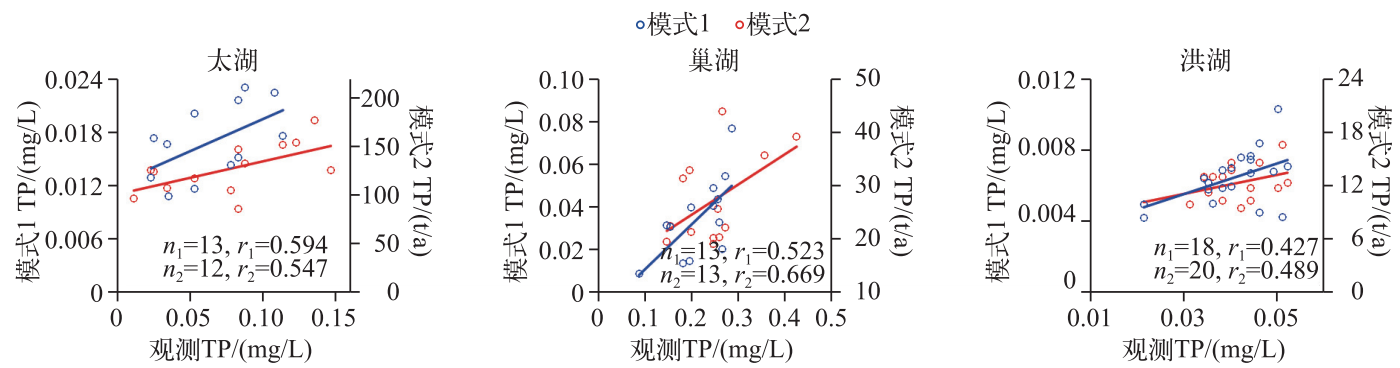

图 3 1950-2000 A.D. 部分湖泊模式 1 和模式 2 总磷模拟值与观测值相关关系

Fig.3 Correlations between the simulated total phosphorus and observed total phosphorus of Lake Taihu, Lake Chaohu and Lake Honghu during 1950 - 2000 A.D.



图 4 模式 1 和模式 2 对 1640-1840 A.D.总磷变化模拟结果(均采用距平进行对比)

Fig.4 Anomalies of simulated total phosphorus of the 8 lakes by Model 1 and Model 2 during 1640 - 1840 A.D.

个湖泊均呈显著相关 $(n=25 \sim 73, P \leqslant 0.1)$ (图 5), 说明模拟重建的湖泊水体营养盐变化与沉积钻孔揭示的 历史营养盐变化基本一致.

\section{5 分析与讨论}

\section{1 湖泊营养态长期演化的区域性特征}

模式 1 模拟的 1640-1840 A.D. 8 个湖泊的营养盐浓度平均值在 $0.03 \sim 0.24 \mathrm{mg} / \mathrm{L}$ 之间变化(图 6), 与 1998-2000 年开展的第二次湖泊调查结果 $(0.035 \sim 0.268 \mathrm{mg} / \mathrm{L})^{[19]}$ 基本一致,除巢湖外,其余 7 个湖泊的平 




图 5 模式 1 和模式 2 总磷浓度变化模拟结果与沉积钻孔总磷含量变化对比

Fig. 5 Correlations of simulated total phosphorus and sediment total phosphorus of the lakes by Model 1 and Model 2

均模拟 TP 浓度均高于现代观测值 ${ }^{[11,19]}$, 可见 1640-1840 A.D. 期间长江中下游湖泊已经处于较高的营养水 平. 从理论上看, 长江中下游湖泊高营养背景值因与这些湖泊所处的洪泛平原有关. 在浅水湖泊中, 湖泊内 的水生动、植物和浮游动、植物的群落组成与水文特性和营养盐资源息息相关. 特别是洪水时期来自河流的 氮、磷输人是水体富营养化的主要渠道. 洪水的一次次泛滥, 将带来大量的营养盐, 沉淀在这些地势低洼的 湖泊和湿地, 使得这些水体中生物量等较没有洪水泛滥的湖泊多得多, 造成营养盐富集. 其次, 浅水湖泊风 浪扰动强烈, 湖底沉积物在风浪作用下发生悬浮, 导致大量的营养盐从沉积物中释放出来并进人上覆水, 并 为生物生长所利用, 因此浅水湖泊內源污染负荷较深水湖泊更重. 第三, 长江中下游湖泊大多数是通江湖 泊, 接受了长江沉积物. 由于湖泊水动力较小, 与江河沉积相比, 沉积在湖泊中的泥沙颗粒相对细小. 细小的 颗粒吸附能力强, 往往吸附大量的营养盐. 经过长时期的积累, 造成湖泊底泥中营养盐含量普遍较高 ${ }^{[37]}$.

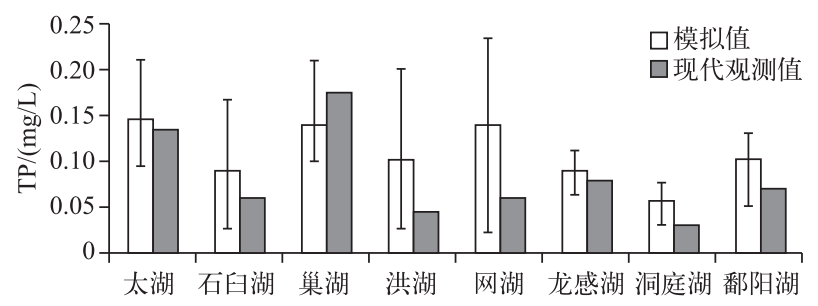

图 6 长江中下游 8 个湖泊的历史总磷浓度与现代观测值比较

Fig. 6 Comparisons between simulated total phosphorus concentration and modern observation data in 8 lakes of the middle and lower reaches of Yangtze River

\section{2 湖泊营养盐变化对气候的响应}

根据 GCM 模拟的 1640-1840 A.D. 气候状况, 从温度与降水逐年变化 20 年滑动平均值看 (图 7a), 温度 与降水同步变化的阶段占 $39 \%$, 异步变化的阶段占 $45 \%, 16 \%$ 的变化阶段不能确定. $1640-1840$ A.D. 正处于 我国明清小冰期时期, 气候特征为冷干, 故分别选取降水和温度极小值出现时期作为本研究的典型气候时 期. 降水最低值出现在 18 世纪中期 (1748-1767 A.D.) , 与湖泊沉积记录揭示的小冰期干冷期一致 ${ }^{[38]}$, 该时 期 (本文称时期 1) 61\% 的降水低于多年平均降水量; 随后在 1776-1795 A.D. 出现温度最低值, 该时期 (时期 2) $58 \%$ 的温度低于多年平均温度,形成典型的湿冷气候期,已有研究证实了该时期的存在 ${ }^{[39]}$.

采用主成分分析 (PCA) 提取模拟的 8 个湖泊营养盐变化的共同特征. 模式 1 中第一和第二主成分的累 积方差贡献率达到 $90.0 \%$, 模式 2 中第一和第二主成分的积累方差贡献率达到 $89.6 \%$, 计算两个主成分的合 
量表征 2 种模式下营养盐的变化,并同样进行 20 年滑动平均处理( 图 7b). 可见,时期 1 也是营养盐变化最 低值出现的时期,在该时期 $20 \%$ 的 TP 变化在 $+0.5 \% \sim+10.6 \%$,而 $60 \%$ 的 TP 变化在 $-36.1 \% \sim-5.1 \%$. 在时期 2 ,模拟的气候结果显示出降水的一个高值时期,此时期与 TP 的高值时期对应. 在该时期, $35 \%$ 的 TP 变化在 $+1.0 \% \sim+18.3 \%$ 之间, $45 \%$ 的 TP 变化在 $-16.9 \% \sim-1.6 \%$ 之间. 说明湖泊营养盐的降低更倾向于受到干旱气 候的影响,而营养盐变化对于寒冷气候的响应并不明显.

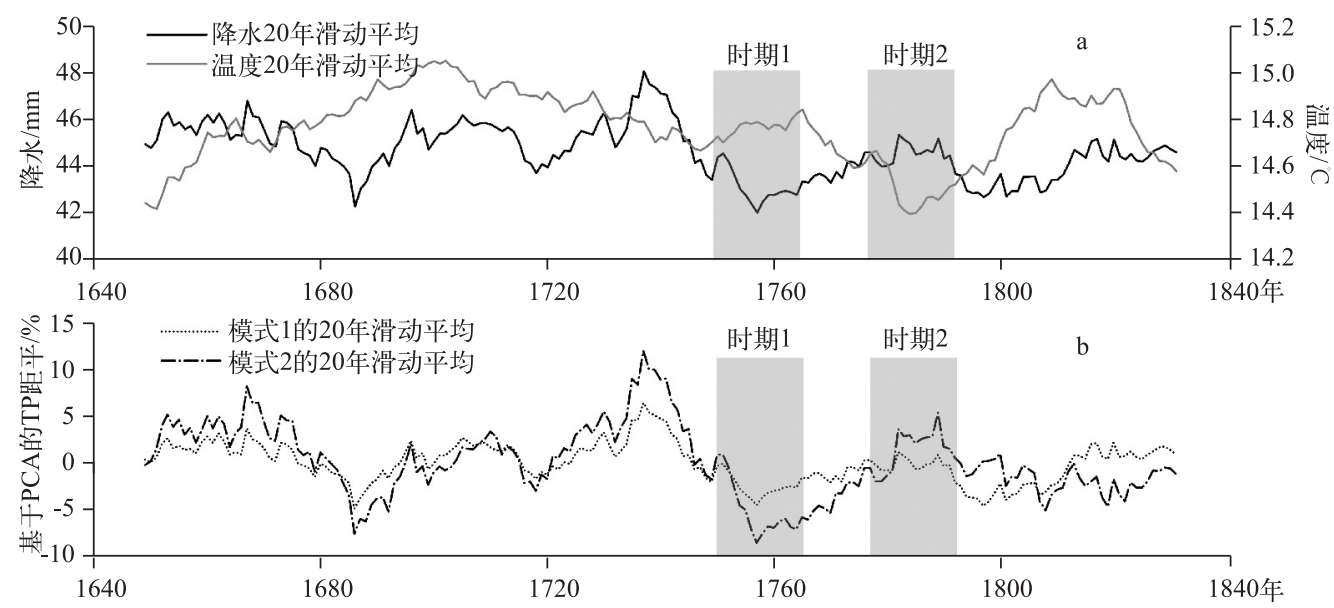

图 7 基于 GCM 模式 1640-1840 A.D.降水和温度 20 年变化滑动平均曲线( (a)及 2 种模式模拟的 TP 距平变化 20 年滑动平均曲线 $(\mathrm{b})$

Fig.7 20-year moving averages of annual precipitation and temperature from GCM model (a) and historical nutrient simulations during 1640 - 1840 A.D. in Model 1 and Model 2(b)

笔者分别将 1640-1840 A.D. 降水、温度序列与 8 个湖泊在 2 种模式下模拟的营养盐变化序列进行相关 性分析. 结果显示, 8 个湖泊营养盐与降水相关系数 $r^{2}$ 高达 $0.78 \sim 0.85$, 而与温度的相关系数 $r^{2}$ 仅为 $0.42 \sim$ 0.53 , 且湖泊容量越小 (如石臼湖、洪湖、网湖、龙感湖等) 其营养盐水平与气候的相关系数越大, 容量越大的 湖泊 (如鄱阳湖、太湖等) 相关系数越小. 说明长江中下游湖泊营养盐变化对气候要素中降水变化响应更敏 感, 降水增多时, 由于强降水冲刷地表和侵蚀土壤, 流域径流增大, 将大量的矿物质和营养盐带人湖泊水体, 从而导致湖泊营养盐浓度上升; 降水减少时, 来自于外源的营养盐减少, 因此湖泊水体营养盐浓度下降. 面 积在 $400 \mathrm{~km}^{2}$ 以下湖泊的营养盐对气候变化的响应较之面积 $2000 \mathrm{~km}^{2}$ 以上的大湖更为敏感.

\section{3 湖泊营养盐历史变化的主导因素识别}

由于 2 个模式具有不同的驱动和反馈因子, 通过对 2 种模式下湖泊营养盐历史变化过程进行比较, 从而 认识营养盐变化机制. 5.2 节从模式的驱动因子差异, 对 2 个模式结果做出气候响应的解析. 下面进一步从 2 个模拟结果的频率分布上, 定量分析气候与 生物量不同因子的贡献量.

模拟结果显示外源模式和内源模式下 湖泊营养盐变化具有一致性, 说明 $1640-$ 1840 A.D. 期间营养盐变化主要受流域营养 盐输人的影响. 通过频率分布情况进一步比 较 8 个湖泊的营养盐平均值在两种模式下 的变率. 图 8 中模式 1 和模式 2 的频率分布 曲线均服从正态分布, 其中模式 1 的标准偏 差为 11.9 ,模式 2 的标准偏差为 20.3 , 可见 模式 2 的离散程度明显高于模式 1 , 说明模

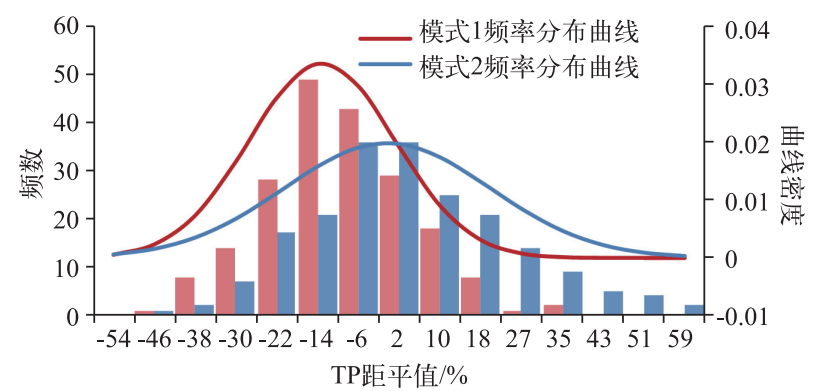

图 82 种模式模拟的营养盐变化频率分布曲线

Fig. 8 Frequency distributions of TP in Model 1 and Model 2 
式 2 由于生态系统的参与, 使营养盐响应外界气候变化具有更大的容量 (包括缓冲和滞后能力). 进一步定 量分析来源于生态系统的营养盐变化的贡献, 模式 1 在 90 th 百分位营养盐变化为 $15.3 \%$, 在 10 th 百分位营 养盐变化为 $-15.5 \%$; 模式 2 在 90 th 百分位营养盐变化为 $27.2 \%$, 在 10 th 百分位营养盐变化为 $-23.8 \%$, 可 见, 在气候和生物量两大因子作用营养盐变化中, 来源于生态系统的营养盐 (湖泊生物积累) 对湖泊营养盐 富集的贡献在标准差中占 $41.4 \%$,在 10 th 90 th 的百分位占 $39.5 \%$.

\section{4 湖泊营养盐历史演变研究的现实意义}

基于本文构建的两种模式, 通过对历史时期 1640-1840 A.D.长江中下游湖泊营养盐历史演变的模拟及 比较阐明了营养盐的外源输人 (流域来源) 是影响湖泊营养盐变化的主导因素, 并认识到生态系统的反馈作 用在营养盐富集中的贡献. 鉴于此,对营养盐外源与内源的湖泊水体修复措施提出论证.

1) 外源控制是保障湖泊水质的根本途径. 近年来一些江河湖泊为防洪防侵蚀, 建立大量的硬质堤坝, 实 质上是切断了陆域生态系统到水域生态系统的连续性, 江河湖生态环境缓冲带消失, 加重了蓝藻水华、供水 危机、生产安全等环境问题. 通过对长江中下游湖泊历史营养盐演变的研究发现, 在气候驱动下, 来自流域 的营养盐输人对湖泊营养盐的长期变化具有主导作用. 因此, 保证湖泊与长江水体的周期交换, 保持从陆域 到水域生态系统的连续性,维护江-湖生态环境的缓冲带, 是实现湖泊水质改善目标的根本途径.

2) 通过生态修复改善湖泊水质的有效性与局限性并存. 当前,一些湖泊出现环境问题后, 首先考虑采用 生态修复措施, 通过对湖泊生物链网进行优化改造, 以恢复生物的多样性和改善水质. 根据本文的模拟结 果, 生态修复确实能起到一定的改善水质的效果, 但必须有外源的污染控制措施与之配套, 才能充分发挥其 作用. 建议在加快湖泊生态的自然恢复进程中, 加强流域污染物输人的控制. 这个理念在短期的湖泊修复已 见成效,本文研究说明了对湖泊长期变化和趋势的控制仍然不失一种有效途径.

致谢: 感谢廖梦娜、李永飞对湖泊生物、营养盐数据集的编辑、数据化等协助工作.

\section{6 参考文献}

[ 1 ] Bergengren JC, Waliser DE, Yung YL. Ecological sensitivity: a biospheric view of climate change. Climatic Change, 2011, 107(3) : 433-457. DOI 10.1007/s10584-011-0065-1.

[ 2 ] Jeppesen E, Kronvang B, Meerhoff M. Climate change effects on runoff, catchment phosphorus loading and lake ecological state, and potential adaptations. Journal of Environmental Quality, 2009, 38 ( 5 ): 1930-1941. DOI 10. 2134/ jeq2008.0113.

[ 3 ] Qin Boqiang, Gao Guang, Zhu Guangwei et al. Lake eutrophication and its ecosystem response. Chinese Science Bulletin, 2013, 58(10) : 961-970( in Chinese with English abstract). DOI 10.1007/s11434-012-5560-x. [秦伯强, 高光, 朱广伟 等. 湖泊富营养化及其生态系统响应. 科学通报, 2013,58 (10): 961-970.]

[ 4 ] Schindler DW. Recent advances in the understanding and management of eutrophication. Limnology and Oceanography, 2006, 51 (1) : 356-363. DOI 10.4319/lo.2006.51.1_part_2.0356.

[ 5 ] Chen Xiaoying, Li Shijie. An analysis on the evolvement processes of lake eutrophication and their characteristics of the typical lakes in the middle and lower reaches of Yangtze River. Chinese Science Bulletin, 2006, 51(7) : 848-855 (in Chinese with English abstract). [ 成小英, 李世杰. 长江中下游典型湖泊富营养化演变过程及其特征分析. 科学通报, $2006,51(7): 848-855$. ]

[ 6 ] Perrow MR, Davy AJ. Handbook of ecological restoration. Cambridge: Cambridge University Press, 2002: 1-80.

[ 7 ] Phillips G, Kelly A, Pitt J et al. The recovery of a very shallow eutrophic lake, 20 years after the control of effluent derived phosphorus. Freshwater Biology, 2005,50 ( 10 ) : 1628-1638. DOI 10.1111/j.1365-2427.2005.01434.x.

[ 8 ] Qin Boqiang, Wu Qinglong, Gao Junfeng et al. Water environmental issues in Taihu Lake of China: Problems, causes and management. Journal of Natural Resources, 2002,17(2): 221-228 (in Chinese with English abstract). [秦伯强, 吴庆 龙, 高俊峰等. 太湖地区的水资源与水环境——问题、原因与管理. 自然资源学报,2002,17(2): 221-228.]

[ 9 ] Liu Enfeng. Geochemistry characteristic of the sediment from typical lakes in middle-lower reaches of Yangtze River and their response to human activities—case studies of Lakes Taihu and Taibaihu[Dissertation]. Nanjing: Nanjing Institute of Geography and Limnology, CAS, 2005 (in Chinese with English abstract). [刘恩峰. 长江中下游典型湖泊沉积物地球 化学特征及人类活动响应——太湖、太白湖为例 [学位论文]. 南京: 中国科学院南京地理与湖泊研究 
所, 2005.]

[10] Barry Saltzman. Dynamical paleoclimatology : generalized theory of global climate change. New York: Academic Press, 2002: 1-35. DOI 10.1002/joc.879.

[11] Wang Suming, Dou Hongshen eds. Chinese Lakes. Beijing: Science Press, 1998: 1-942(in Chinese). [王苏民, 窦鸿 身. 中国湖泊志. 北京: 科学出版社, 1998: 1-942.]

[12] Yang Dayuan, Li Xusheng, Zhang Zhenke. Lake evolution along middle-lower reaches of the Yangtze River. J Lake Sci, 2000, 12(3) : 226-232 (in Chinese with English abstract). DOI 10. 18307/2000. 0306. [杨达源, 李徐生, 张振克. 长 江中下游湖泊的成因与演化. 湖泊科学, 2000,12(3): 226-232. ]

[13] Qin Boqiang. Lake Taihu, China-Dynamic and environment changes. Netherlands: Springer, 2008: 229-292.

[14] Yu Ge, Guo Ya, Liao Mengna. Probe on attributions of extreme floods responding to the climate changes in Lake Taihu catchment. J Lake Sci, 2013,25(5) : 765-774( in Chinese with English abstract). DOI 10.18307/2013.0520. [于革, 郭 娅, 廖梦娜. 气候变暖下太湖极端洪水的归因探讨. 湖泊科学, 2013,25(5): 765-774.]

[15] Thornthwaite CW. An approach towards a rational classification of climate. Geography Rev, 1948,38 (1) : 55-94. DOI 10. $2307 / 210739$.

[16] Yu Ge, Liao Mengna, Li Yongfei. Response of lake ecosystem to the climate changes of the past 1000 years. Quaternary Sciences, 2013, 33(6) : 1148-1159(in Chinese with English abstract). DOI 10.3969/j.issn.1001-7410.2013.06.11. [于 革, 廖梦娜, 李永飞. 湖泊生态系统对过去 1000 年气候变化的响应研究. 第四纪研究, 2013,33 (6): 1148-1159.]

[17] LÜ Lanjun. Analysis and evaluation of the condition of the water quality of the Poyang Lake. Yangtze River, 1992, 23( 7) : 17-24 (in Chinese with English abstract). [吕兰军. 鄱阳湖水质状况分析与评价. 人民长江, 1992, 23 (7): 17-24.]

[18] Han Xiaoyong. Investigation and research on the water quality of Lake Chaohu. Water Resource Protect, 1998, 1(3) : 24-28 (in Chinese with English abstract). [ 韩小勇. 巢湖水质调查与研究. 水资源保护, 1998,1(3): 24-28.]

[19] Yang Guishan, Ma Ronghua, Zhang Lu et al. Lake status major problems and protection strategy in China. J Lake Sci, 2010, 22(6) : 799-810( in Chinese with English abstract). DOI 10. 18307/2010. 0601. [杨桂山, 马荣华, 张路等. 中 国湖泊现状及面临的重大问题与保护策略. 湖泊科学,2010, 22(6): 799-810.]

[20] Yao Shuchun, Xue Bin, Xia Weilan. Human impact recorded in the sediment of Honghu Lake. Resources and Environment in the Yangzte Basin, 2005,14 (4) : 475-480(in Chinese with English abstract). [姚书春, 薛滨, 夏威岗. 洪湖历史时 期人类活动的湖泊沉积环境响应. 长江流域资源与环境, 2005，14 (4) : 475-480.]

[21] Yao SC, Xue B, Kong DY. Chronology and nutrients change in recent sediment of Taihu Lake, lower Changjiang river basin, East China. Chinese Geographical Science, 2010, 20(3) : 202-208. DOI 10.1007/s11769-010-0202-1.

[22] Yao Shuchun, Xue Bin. Recent environmental evolution of Shijiuhu Lake inferred from lake sediments. Quaternary Sciences, 2009, 29(2) : 248-255 (in Chinese with English abstract). [姚书春, 薛滨. 石臼湖近代环境演化历史. 第四纪 研究, 2009, 29 (2) : 248-255.]

[23] Liu Enfeng, Du Chengchang, Yang Xiangdong et al. Enrichment characteristic of phosphorus in surface and core sediments of Chaohu Lake and the pollution quantification. Environmental Science, 2012, 33 (9) : 3024-3030 (in Chinese with English abstract). [刘恩峰, 杜臣昌, 羊向东等. 巢湖沉积物中磷蓄积时空变化及人为污染定量评价. 环境科学, $2012,33(9)$ : 3024-3030.]

[24] Yin Faneng. Research on natural environment evolution of the Honghu Lake. Yangtze River, 2008, 39(5): 19-23 (in Chinese with English abstract). [尹发能. 洪湖自然环境演变研究. 人民长江, 2008, 39 (5): 19-23. ]

[25] Shen Ji, Xue Bin, Wu Jinglu et al eds. Lake sediments and environmental evolution. Beijing: Science Press, 2010 : $257-$ 326(in Chinese). [沈吉,薛滨,吴敬禄等. 湖泊沉积与环境演化. 北京: 科学出版社, 2010: 257-326.]

[26] Shi Xiaoli, Qin Boqiang. Evolution and ecological environment of lakes in the middle and lower reaches of Yangtze River. Journal of Ningbo University (NSEE), 2007, 20 (2) : 221-227(in Chinese with English abstract). [史小丽, 秦伯强. 长 江中下游地区湖泊的演化及生态特性. 宁波大学学报, 2007, 20(2) : 221-227.]

[27] Wu Yanhong, Wang Sumin. Estimate of anthropogenic nutrient element fluxes recorded in lacustrine sediments: a case study in Longgan Lake. Quaternary Sciences, 2006, 26(5) : 843-848(in Chinese with English abstract). [ 吴艳宏, 王苏 民. 龙感湖沉积物中人类活动导致的营养盐累积通量估算. 第四纪研究, 2006, 26 (5) : 843-848.]

[28] Liu Jian, Yang Xiangdong, Wang Sumin. Study on the nutrient evolution and its controlling factors of Longgan Lake for the last 200 year. Science China: Series D, 2005, 35(S II ) : 173-179( in Chinese with English abstract). [刘健, 羊向东, 
王苏民. 近两百年来龙感湖营养演化及其控制因子研究. 中国科学: D 辑, 2005, 35(增刊 II )：173-179.]

[29] Yang Xiangdong, Shen Ji, Dong Xuhui et al. Historical trophic evolutions and their ecological responses from shallow lakes in the middle and lower reaches of the Yangtze River: case studies on Longgan Lake and Taibai Lake. Science China: Series $D, 2005,35$ ( S II ) : 45-54 (in Chinese with English abstract). [羊向东, 沈吉, 董旭辉等. 长江中下游浅水湖泊历史 时期营养态演化及其与水生生态系统的关系——龙感湖、太白湖为例. 中国科学: D 辑, 2005, 35(增刊 II ) : 45-54.]

[30] Dingman SL. Physical hydrology, 2nd edition. Prentice Hall, Upper Saddle River, NJ, 2002: 82-492.

[31] Wetzel RG. Limnology: lake and river ecosystem, 3rd edition. San Diego, CA: Academic Press, 2001: 129-149.

[32] Xie Ping ed. Scaling ecology to understand natural design of life systems and their operations and evolutions. Beijing: Science Press, 2013: 397 (in Chinese). [谢平. 从生态学透视生命系统的设计、运作与演化一一生态、遗传和进化通过 生殖的融合. 北京: 科学出版社, 2013: 397. ]

[33] Jesser I. The influence of macrophytes on a phytoplankton community in experimental conditions. Hydrobiologia, 1995, 306 (1) : 21-32. DOI 10.1007/BF00007855.

[34] Tang Jingbo, Hu Zhiyuan, Zhang Yanqiong. Determination of high accuracy parameters in Lotka-Volterra artificial ecosystem model. Mathematics in Practice and Theory, 2007,37 (14): 77-82(in Chinese with English abstract). [唐静波, 胡 智渊, 张彦琼. 确定 Lotka-Volterra 生态系统模型高精度参数的研究. 数学的实践与认识, 2007,37 (14) : 77-82.]

[35] Kong Fanxiang, Song Lirong eds. Research on forming process and environmental characteristics of cyanobacteria bloom. Beijing: Science Press, 2011: 1-40(in Chinese). [ 孔繁翔, 宋立荣. 蓝藻水华形成过程及其环境特征研究. 北京: 科学出版社, 2011: 1-40.]

[36] Ren Junlong, Liu Cunqi, Tian Zhifu et al. Linear analysis on growth parameters of Potamogeton crispus in Baiyangdian Lake. Journal of Hebei University: Natural Science Edition, 2012, 32 (2) : 187-192(in Chinese with English abstract). [任俊龙, 刘存歧, 田志富等. 白洋淀菹草生长参数的线性分析. 河北大学学报: 自然科学版, 2012, 32 (2): 187-192.]

[37] Qin Boqiang, Yang Liuyan, Chen Feizhou et al. Mechanism and control of lake eutrophication. Chinese Science Bulletin, 2006, 51(16) : 1857-1866(in Chinese with English abstract). [秦伯强,杨柳燕,陈非洲等. 湖泊富营养化发生机制 与控制技术及其应用. 科学通报, 2006,51(16): 1857-1866.]

[38] Jin Zhangdong, Wang Sumin, Shen Ji et al. Weak chemical weathering during the Little Ice Age recorded by lake sediments. Science China: Series D, 2001, 31(3): 652-658(in Chinese with English abstract). [ 金章东, 王苏民, 沈吉等. 小冰期弱化学风化的湖泊沉积记录. 中国科学: D 辑, 2001, 31 (3) : 652-658.]

[39] Li Mingqi, Jin Helin, Zhang Hong. Advances of climate research in the Little Ice Age. Journal of Desert Research, 2005, 25 (5) : 731-737 (in Chinese with English abstract). [李明启, 靳鹤龄, 张洪. 小冰期气候的研究进展. 中国沙漠, $2005,25(5): 731-737$. 